\title{
Revascularization of Left Anterior Descending Coronary Artery Chronic Total Occlusion by Percutaneous Coronary Intervention versus Coronary Artery Bypass Grafting (Early and Mid Term Outcome)
}

\author{
Dr Ahmed Boghdady \\ Assistant Professor, Sohag University Hospital, \\ Internal Medicine Department, Egypt \\ ahbomed@gmail.com \\ Dr Ali Kasem \\ Professor, Sohag University Hospital, \\ Internal Medicine Department, Egypt \\ alikasse@gmail.com
}

\author{
Dr Sharaf Eldeen Shazly Abdalla
}

Lecturer, Sohag University Hospital, Internal Medicine Department, Egypt sharaf003@gmail.com

\section{Dr Mohamed Eid}

Lecturer, Sohag University Hospital, Internal Medicine Department, Egypt mhmdeid2008@yahoo.com

\begin{abstract}
:
Background In spite of continuing progress in percutaneous coronary intervention (PCI), recanalization of CTO still remains a challenge for invasive cardiologists. Yet recent advances in PCI materials, devices, approaches, and techniques have allowed expert operators to successfully tackle complex cases of CTO that many years ago have been sent patients to coronary artery bypass surgery (CABG). A comparison between revascularization of isolated left anterior descending (LAD) artery CTO through PCI and that by coronary artery bypass grafting may not be previously studied. Aim of the work To evaluate short and intermediate term outcome of revascularization of LAD CTO in symptomatic patients by PCI and to compare this outcome with $\angle A D C T O$ revascularization through $C A B G$.
\end{abstract}

Patients and methods: A prospective single center observational study. The present study was performed on 80 patients with LAD CTO identified at coronary angiography of Sohag Cathlab of Internal Medicine Department, Sohag University Hospital during the period from January 2012 to March 2013. All patients had a native vessel total occlusion of LAD of at least three months duration based on a history of sudden chest pain, a previous MI in the same target vessel territory, or the time between diagnosis made on coronary angiography and PCI. All patients had symptomatic angina and/or a positive functional study for ischemic heart disease (Thallium 201 radionuclide perfusion imaging). Patients were divided into 2 groups according to coronary angiography in which Group 1: include 40 patients underwent successful PCI. Group 2: include 40 patients underwent CABG.

Results: During the period of follow up ( 12 months), total MACE ( major adverse cardiac events) was more common in PCI group (10 patients, 25\%) than in CABG group (6 patients, 15\%, P=0.049) but there was no significant difference in death (2[5\%] in PCI vs 2 [5\%] in CABG), MI (2 [5\%] in PCI vs (1[2.5\%] in CABG) or stroke (1[2.5\%] in PCI vs 1 [2.5\%] in CABG) between both groups but repeat revascularization was not significantly higher in PCI group (5 patients, 12.5\%) than CABG group (2 patient, 5\%, P=0.083). Summary and Conclusion: Our findings suggest that $C A B G$ remained a superior intervention for patients with isolated $L A D$ CTO lesion than PCI. The most benefit of CABG was to reduce the odds of repeat revascularization as compared with PCI in a short-term follow-up.

\section{INTRODUCTION}

Chronic total occlusion (CTO) of coronary arteries is defined as complete occlusion of coronary vessel with TIMI 0 flow greater than 3 months. ${ }^{1}$ Histopathologically, CTOs are characterized by fibrous caps, varying degrees of plaques, and neovascularization, with direct relationship with advancing age of arising and appearance of these disorders. 2 The exact incidence CTOs during routine coronary angiography is not well defined. ${ }^{3}$ Angioplasty for this coronary lesion has been avoided for many years, and the guideline suggested that single vessel disease being medically treated and multivessel disease for surgical intervention mainly because of the low success rate of percutaneous coronary interventions (PCI) and high restenosis rates following PCI. ${ }^{4}$ In spite of 
continuing progress in PCI, recanalization of CTO remains a challenge for invasive cardiologists. 5 Yet recent advances in PCI materials, devices, approaches, and techniques have allowed expert operators to successfully tackle complex cases of CTO. ${ }^{6}$ A comparison between revascularization of isolated LAD coronary CTO through PCI (percutaneous coronary intervention) or coronary artery bypass grafting may not be previously studied.

\section{AIM OF THE WORK}

To evaluate short term outcome of revascularization of LAD CTO in symptomatic patients by PCI and compare with LAD CTO revascularization through CABG.

\section{Patients And Methods}

A prospective single center observational study. The present study included patients with LAD CTO identified at coronary angiography of Sohag Cathlab of Internal Medicine Department, Sohag University Hospital during the period from 1/1/2012 to 1/3/2013 who agreed to participate in this study and signed an informed consent. The study has been approved from Ethical Review Committee at Sohag Faculty of Medicine. All patients had a native vessel total occlusion of LAD of at least three months duration based on a history of sudden chest pain, a previous myocardial infarction (MI) in the same target vessel territory, or the time between diagnosis made on coronary angiography and PCI. All patients had symptomatic angina and/or a positive functional study for ischemic heart disease (Thallium 201 radionuclide perfusion imaging).

Inclusion criteria: 1-Patients with symptomatic ischemic heart disease (stable angina, unstable angina) amenable for PCI for CTO of LAD with favorable angiographic characteristics(Short segment, mild to moderate calcification, absence of side branch, absence of bridging collaterals, Presence of tapered stump). 2-Patients with symptomatic ischemic heart disease (stable angina, unstable angina) amenable for CABG for CTO of LAD with unfavorable angiographic characteristics (Long segment, heavy calcification, presence of side branch or more, presence of bridging collaterals, absence of tapered stump, failed recanalization through PCI).3-Elective PCI or CABG. 4-Age >18 years.

Exclusion criteria: 1-Emergency PCI (e.g. Primary PCI, Rescue PCI) or emergency CABG.2-Non LAD CTO.3-Contraindication to cardiac catheterization(A- Coagulation Disorder or hepatic failure. B- Renal insufficiency (serum creatinine $>2 \mathrm{mg} / \mathrm{dl}$ ). C- Allergy to contrast medium. D-Patient refusal to receive invasive treatment. 4- Severe left ventricular (LV) dysfunction (ejection fraction (EF) $<30 \%)$. 5-Three vessels disease (3VD) and left main disease (LMD) 6-Pregnancy, congenital or rheumatic heart disease, and malignancy.7-asymptomatic patient with negative perfusion imaging suggestive of ischemia at LAD territory (scar).

\section{Method}

Patients were divided into 2 groups according to type of procedure. Group 1: included 40 patients with successful PCI. Group 2: included 40 patients with CABG.

All patients are subjected to: - 1-Full history taking and examination to assess: Cardiovascular risk factors and symptoms suggestive of coronary insufficiency especially typical effort anginal pain and the impact of symptoms on patient functional capacity. Every patient was assessed as regards the Canadian Cardiovascular Society classification of Angina (CCS) class, Also every patient was assessed as regards the NYHA classification of heart failure. 2-Laboratory investigations; including complete blood picture, fasting blood glucose, serum lipid profile, serum creatinine, liver function tests, and serology for hepatitis B and C. 3- 12 lead surface ECG to assess and identify: Classic ECG criteria of ischemia, and prior MI.

4-The Echocardiography were performed with Toshiba instruments, Japan (Nemio SSA-550A), with a 2.5-MHz transducer and harmonic imaging at the Internal Medicine Department Echocardiography Laboratory. All echocardiography results were obtained before, 6 and 12 month after revascularization. Left ventricular systolic (LVSD) and diastolic diameters (LVDD) are measured by M-mode echocardiography. Left ventricular ejection fraction (LVEF) is assessed using the modified biplane Simpson's method. Mitral regurgitation (MR) is graded with color flow imaging. MR is considered mild when the regurgitated jet area occupied 5-20\% of the LA area, moderate when it occupied $20-40 \%$ and severe when it occupied more than $40 \%$ of the LA area. 7 The LV systolic 
Revascularization of Left Anterior Descending Coronary Artery Chronic Total Occlusion by Percutaneous Coronary Intervention versus Coronary Artery Bypass Grafting (Early and Mid Term Outcome)

function is classified by ejection fraction (EF) from grade 1 to grade 4 according to (Lang et al, 2006). 8

5- Coronary angiography performed using Toshiba instrument (Biplane Infinix CB, Japan) (Seldinger's technique). The procedures and lesions identifications are performed by expert interventional cardiologists.

Preprocedural preparation: Informed consent: A detailed discussion with the patient and family for indications, benefits, potential complications; possibility to need emergency CABG as well as alternative options then an informed consent was taken. All medications are continued till morning of the procedure. Aspirin $325 \mathrm{mg}$ orally is given before the procedure. If the patient is not on Clopidogril $75 \mathrm{mg}$, a loading dose of $600 \mathrm{mg}$ PO is given prior to the procedure. Continuous blood pressure \& ECG monitoring during the whole procedure were performed. Resuscitation equipment, in particular defibrillators \& intubation trays, are always tested \& ready. Viability studies are done in patients with marked wall motion abnormalities in LAD territory to detect viable myocardium before PCI.

PCI procedure: Access site: Transfemoral standard technique is used in all patients using $6 \mathrm{Fr}$ sheath. Coronary angiography is performed assessing CTO lesion as regards: site of the occlusion, length of the occlusion, if calcified, degree of calcification. Also detection of collaterals using Rentrop grade. ${ }^{\mathbf{9}}$ analysis of TIMI flow grade, Grade $\mathbf{0}=$ no visible filling. Grade $\mathbf{1}=$ side branch filling but without any contrast medium reaching the epicardial segment. Grade $\mathbf{2}=$ partial epicardial filling. Grade 3 = complete filling of the epicardial segment.

For the left coronary system, extra backup type guiding catheters (extra backup) were preferred and used in most cases. Judkins-type guiding catheters were not used frequently as they typically preclude deep intubation \& associated with reduced success with hard fibrocalcific occlusions. Antegrade approach to CTO is the strategy applied in all patients of our study. Contra lateral injection was used in few patients.

\section{DEFINITIONS}

1-Technical success was a successful recanalization and dilation of CTO with a residual stenos is of < $20 \%$, and TIMI flow $>2$ as assessed by quantitative coronary angiography. 10 2-Procedural successes was a technical success with no in hospital MACE. 103-MACE was defined as death, MI, stroke and target lesion revascularization (TLR). 10 4-TLR any repeat percutaneous intervention of the target lesion (including $5 \mathrm{~mm}$ proximal and distal to the target lesion) or surgical bypass of the target vessel performed for restenosis or other complication involving the target lesion. $\mathbf{1 1}_{5-\text { In-stent restenosis: is }}$ loss of $50 \%$ or more of the diameter of the in-stent lumen at the site of an initially successful intervention.11 6-Stent Thrombosis is adjudicated according to the Academic Research Consortium (ARC) definitions as definite or probable and by timing of the event: acute ( $\leq 1$ day post-procedure), subacute ( 2 to $\leq 30$ days), late ( $>30$ to $\leq 365$ days), and very late ( $>365$ days). 127-In hospital mortality is a death within the same hospital admission regardless of cause after revascularization. 138- PCI and CABG related MI is arbitrarily defined by elevation of cTn values $(>5 \times 99$ th percentile URL) in patients with normal baseline values ( $\leq 99$ th percentile URL) or a rise of cTn values $>20 \%$ if the baseline values are elevated and are stable or falling. In addition, either (i) symptoms suggestive of myocardial ischemia or (ii) new ischemic ECG changes or (iii) angiographic findings consistent with a procedural complication or (iv) imaging demonstration of new loss of viable myocardium or new regional wall motion abnormality are required. ${ }^{14}$ - Significant CAD was defined as lesion on angiography $\geq 70 \%$ diameter narrowing $\left(\geq 50 \%\right.$ for left main). ${ }^{15}$

\section{Coronary Artery Bypass Graft (CABG)}

CABG was performed using extracorporeal cardiopulmonary bypass (CPB). This included a median sternotomy, internal mammary artery (IMA) dissection, and, when appropriate, simultaneous harvesting of the venous and/or radial artery grafts. The most frequently grafted coronary arteries were the epicardial vessels. The potency of a constructed graft was influenced by characteristics of the anastomosed vessel, the outflow area, the graft material, its manipulation and construction. Important 
coronary characteristics included the internal lumen size, the severity of proximal stenosis, the quality of the wall at the site of anastomosis, and the distal vascular bed. Left internal mammary artery (LIMA) was used for grafting LAD while saphenous vein graft (SVG) was used for LCX or RCA lesions.

\subsection{Both Groups Were Followed Up For One Year and Assessed Outcomes (At 6months and At 12 Months)}

1- Each patient was assessed to changes in class of angina pre and post procedure period.2- Each patient was assessed to changes in class of HF according to NYHA Functional Classification.3Electrocardiogram was performed for every patient. 4-Echocardiography was performed to assess LV dimensions, EF, and degree of MR.5-Coronary angiography was performed .5- MACE was followed before 6 months if patient was symptomatic additionally to regular follow-up at 6 and 12 months..

Statistical analysis: Statistical package for social sciences (IBM-SPSS), version 19 IBM- Chicago, USA was used for statistical data analysis. Data were expressed as mean, standard deviation (SD), number and percentage. Mean and standard deviations were used as descriptive value for quantitative data. Student t test was used to compare the means between two groups, and one-way analysis of variance (ANOVA) test was used to compare means of more than two groups. P-value $<0.05$ was considered as significant.

\section{RESULTS}

Totally, there were 80 patients with LAD CTO at angiography with or without other significant lesions in another coronary artery (left circumflex and/or right coronary artery).. They were divided into 2 groups:

Group 1: includes 40 patients revascularized by PCI.

Group 2: includes 40 patients revascularized by CABG (36 patient immediately after coronary angiography and 4 patients after failure of $\mathrm{PCI}$ ).

The lesion in the other coronary artery was either treated in the same session (if opening CTO took short time or small amount of dye) or at 4 weeks from the same session (if opening CTO took long time or excess contrast)

Table (1) showed that both groups were matched as regard to CV risk factors

Table1. Comparison between both groups as regard CV risk factors

\begin{tabular}{|l|l|l|l|}
\hline Variables & $\begin{array}{l}\text { CABG } \\
\text { N=40 }\end{array}$ & $\begin{array}{l}\text { PCI } \\
\text { N=40 }\end{array}$ & P value \\
\hline $\begin{array}{l}\text { Hypertension } \\
\text { Yes }\end{array}$ & $20(50 \%)$ & $17(42.5 \%)$ & \\
No & $20(50 \%)$ & $23(57.5 \%)$ & 0.083 \\
\hline $\begin{array}{l}\text { Dyslipidemia } \\
\text { Yes } \\
\text { No }\end{array}$ & $21(52.5 \%)$ & $18(45 \%)$ & N.S \\
\hline $\begin{array}{l}\text { DM } \\
\text { Yes }\end{array}$ & $19(47.5 \%)$ & $22(55 \%)$ & 0.083 \\
No & $15(37.5 \%)$ & $13(32.5 \%)$ & N.S \\
\hline $\begin{array}{l}\text { Smoking } \\
\text { Yes }\end{array}$ & $25(62.5 \%)$ & $27(67.5 \%)$ & 0.159 \\
No & $13(32.5 \%)$ & $11(27.5 \%)$ & N.S \\
\hline $\begin{array}{l}\text { Family History } \\
\text { Positive }\end{array}$ Negative & $27(67.5 \%)$ & $29(72.5 \%)$ & 0.323 \\
\hline $\begin{array}{l}\text { Prior MI } \\
\text { Yes }\end{array}$ & $8(20 \%)$ & $7(17.5 \%)$ & N.S \\
No & $32(80 \%)$ & $33(82.5 \%)$ & 0.323 \\
\hline $\begin{array}{l}\text { Prior UA } \\
\text { Yes } \\
\text { No }\end{array}$ & $13(32.5 \%)$ & $12(30 \%)$ & N.S \\
\hline $\begin{array}{l}\text { BMI } \\
<30\end{array}$ & $27(67.5 \%)$ & $28(70 \%)$ & 0.323 \\
$\geq 30$ & $14(35 \%)$ & $16(40 \%)$ & N.S \\
\hline
\end{tabular}

N.S: non significant. 
Revascularization of Left Anterior Descending Coronary Artery Chronic Total Occlusion by Percutaneous Coronary Intervention versus Coronary Artery Bypass Grafting (Early and Mid Term Outcome)

\subsection{Baseline Symptoms and ECG}

There was no statistically significant difference between the two groups as regard to baseline symptoms (Anginal class (CCS) and Dyspnea grade (NYHA) table (2) the same goes for ECG findings table (3).

Table2. Comparison between both groups as regard baseline symptoms

\begin{tabular}{|c|c|c|c|}
\hline Variables & $\begin{array}{l}\text { CABG } \\
\mathrm{N}=40\end{array}$ & $\begin{array}{l}\text { PCI } \\
\mathrm{N}=40\end{array}$ & $P$ value \\
\hline $\begin{array}{l}\text { Angina class }(\mathrm{CCS}) \\
\text { I } \\
\text { II } \\
\text { III } \\
\text { IV }\end{array}$ & $\begin{array}{l}2(5 \%) \\
20(50 \%) \\
14(35 \%) \\
4(10 \%) \\
\end{array}$ & $\begin{array}{l}1(2.5 \%) \\
19(47.5 \%) \\
17(42.5 \%) \\
3(7.5 \%)\end{array}$ & 0.988 \\
\hline $\begin{array}{l}\text { Dyspnea grade (NYHA) } \\
\text { I } \\
\text { II } \\
\text { III } \\
\text { IV }\end{array}$ & $\begin{array}{l}1(2.5 \%) \\
27(67.5 \%) \\
11(27.5 \%) \\
1(2.5 \%)\end{array}$ & $\begin{array}{l}1(2.5 \%) \\
23(57.5 \%) \\
13(32.5 \%) \\
3(7.5 \%)\end{array}$ & 0.808 \\
\hline
\end{tabular}

Table3. Comparison between both groups as regard ECG findings

\begin{tabular}{|l|l|l|l|}
\hline ECG findings & CABG $(\mathrm{N}=40)$ & PCI $(\mathrm{N}=40)$ & P value \\
\hline Old anterior MI & $10(25 \%)$ & $8(20 \%)$ & 0.592 \\
\hline Old inferior MI & $3(7.5 \%)$ & $4(10 \%)$ & 0.999 \\
\hline ST depression \&T inversion & $13(32.5 \%)$ & $14(35 \%)$ & 0.813 \\
\hline LBBB & $7(17.5 \%)$ & $8(20 \%)$ & 0.775 \\
\hline Normal & $7(17.5 \%)$ & $6(15 \%)$ & 0.762 \\
\hline
\end{tabular}

\subsection{Angiographic Data}

Angiographic data are shown in table (4) with No significant difference between both groups.

Table4. Comparison between both groups as regard angiographic data

\begin{tabular}{|l|l|l|l|}
\hline Diseased artery & $\begin{array}{l}\text { CABG } \\
\text { N=40 }\end{array}$ & $\begin{array}{l}\text { PCI } \\
\text { N=40 }\end{array}$ & P value \\
\hline LAD & $40(100 \%)$ & $40(100 \%$ & \\
\hline LCX & $10(25 \%)$ & $7(17.5 \%)$ & 0.354 \\
\hline RCA & $6(15 \%)$ & $4(10 \%)$ & 0.520 \\
\hline Coronary anatomy & & & \multirow{2}{*}{0.09} \\
1VD & $24(60 \%)$ & $29(72.5 \%)$ & \\
2VD & $16(40 \%)$ & $11(27.5 \%)$ & 0.06 \\
\hline LAD CTO segment & $11(27.5 \%)$ & $7(17.5 \%)$ & \\
Prox: & $29(72.5 \%)$ & $33(82.5 \%)$ & \\
Mid: & & \\
\hline
\end{tabular}

\subsection{Baseline Echocardiographic Data}

No significant difference between both groups as regard baseline echocardiohraphic data as in table (5)

Table5. Comparison between both groups as regard baseline echocardiographic data

\begin{tabular}{|l|l|l|l|}
\hline Variables & $\begin{array}{l}\text { CABG } \\
\text { N=40 }\end{array}$ & $\begin{array}{l}\text { PCI } \\
\text { N=40 }\end{array}$ & P value \\
\hline $\begin{array}{l}\text { LVESD }(\mathrm{mm}) \\
\text { Mean } \pm \text { SD }\end{array}$ & $35.4 \pm 4.64$ & $34.05 \pm 5.652$ & \\
\hline $\begin{array}{l}\text { LVEDD (mm) } \\
\text { Mean } \pm \text { SD }\end{array}$ & $52.85 \pm 4.423$ & $52.72 \pm 4.888$ & 0.247 \\
\hline EF $(\%)$ & & & \\
Mean \pm SD & $50.97 \pm 5.753$ & $51.75 \pm 7.615$ & 0.905 \\
\hline LV grade & & & \\
Grade 1 (EF $\geq 55 \%)$ & $11(27.5 \%)$ & $15(37.5 \%)$ & \\
Grade 2 (EF 40-54\%) & $27(67.5 \%)$ & $22(55 \%)$ & 0.609 \\
Grade 3 (EF 30-39\%) & $2(5 \%)$ & $3(7.5 \%)$ & \\
Grade 4 (EF $<30 \%)$ & $0(0 \%)$ & $0(0 \%)$ & \\
\hline MR degree & & $17(42.5 \%)$ & \\
0 no or minimal & $19(47.5 \%)$ & $15(37.5 \%)$ & 0.996 \\
1 mild & $13(32.5 \%)$ & $8(20 \%)$ & \\
2 moderate & $7(17.5 \%)$ & $0(0 \%)$ & \\
3 severe & $1(2.5 \%)$ &
\end{tabular}


Dr Ahmed Boghdady et al.

\section{Follow Up Results}

\subsection{Angina Pectoris CCS class}

\section{PCI group}

After 6 and 12 months follow up, anginal symptoms were significantly improved; $\mathrm{P}<0.0001$. as shown in Table (6) and figure (1), 2 patients died at 6 month follow up.

\section{CABG group}

There was highly significant improvement in anginal symptoms at $6 \mathrm{~m}$ and $12 \mathrm{~m}$ follow up. $\mathrm{P}<0.0001$ as shown Table (6) with 1 patient died at 6 month FU and another patient at 12 months FU. No significant difference between two groups

\subsection{Dyspnea Grade (NYHA Class)}

There was highly significant improvement in NYHA class after PCI; P<0.0001 and after CABG; P $<0.0001$ but no significant difference between two groups as shown in Table (6) and figure (2).

Table6. Follow up (FU) angina CCS and dyspnea grade (NYHA class) at $6 m$ \& 12 in both groups

\begin{tabular}{|c|c|c|c|}
\hline Variables & $\begin{array}{l}\text { CABG } \\
\mathrm{N}=40\end{array}$ & $\begin{array}{l}\mathrm{PCI} \\
\mathrm{N}=40\end{array}$ & $\mathrm{P}$ value \\
\hline $\begin{array}{l}\text { Angina class (preprocedure) } \\
\text { I } \\
\text { II } \\
\text { III } \\
\text { IV }\end{array}$ & $\begin{array}{l}2(5 \%) \\
20(50 \%) \\
14(35 \%) \\
4(10 \%)\end{array}$ & $\begin{array}{l}1(2.5 \%) \\
19(47.5 \%) \\
17(42.5 \%) \\
3(7.5 \%)\end{array}$ & 0.988 \\
\hline \multirow[b]{2}{*}{$\begin{array}{l}\text { Angina } 6 \mathrm{~m} \mathrm{FU} \\
\text { I } \\
\text { II } \\
\text { III } \\
\text { IV }\end{array}$} & $\begin{array}{c}\text { CABG } \\
\mathrm{N}=39\end{array}$ & $\begin{array}{l}\mathrm{PCI} \\
\mathrm{N}=39\end{array}$ & \multirow[b]{2}{*}{0.996} \\
\hline & $\begin{array}{c}22(56.41 \%) \\
14(35.89 \%) \\
3(7.69 \%) \\
0(0.00 \%) \\
\end{array}$ & $\begin{array}{l}20(51.28 \%) \\
16(41.02 \%) \\
2(5.12 \%) \\
1(2.56 \%) \\
\end{array}$ & \\
\hline & $\begin{array}{l}\text { CABG } \\
\mathrm{N}=38\end{array}$ & $\begin{array}{l}\mathrm{PCI} \\
\mathrm{N}=38\end{array}$ & \multirow[b]{2}{*}{0.990} \\
\hline $\begin{array}{l}\text { Angina } 12 \mathrm{~m} \mathrm{FU} \\
\text { I } \\
\text { II } \\
\text { III } \\
\text { IV }\end{array}$ & $\begin{array}{l}26(68.42 \%) \\
11(28.94 \%) \\
1(2.63 \%) \\
0(0 \%) \\
\end{array}$ & $\begin{array}{l}24(63.15 \%) \\
12(31.57 \%) \\
2(5.26 \%) \\
0(0 \%)\end{array}$ & \\
\hline P1 & $<0.0001$ & $<0.0001$ & \\
\hline $\mathrm{P} 2$ & $<0.0001$ & $<0.0001$ & \\
\hline $\begin{array}{l}\text { NYHA class (preprocedure) } \\
\text { I } \\
\text { II } \\
\text { III } \\
\text { IV }\end{array}$ & $\begin{array}{l}1(2.5 \%) \\
27(67.5 \%) \\
11(27.5 \%) \\
1(2.5 \%)\end{array}$ & $\begin{array}{l}1(2.5 \%) \\
23(57.5 \%) \\
13(32.5 \%) \\
3(7.5 \%)\end{array}$ & 0.808 \\
\hline & $\begin{array}{l}\text { CABG } \\
\mathrm{N}=39\end{array}$ & $\begin{array}{l}\text { PCI } \\
\mathrm{N}=39\end{array}$ & \multirow[b]{2}{*}{0.965} \\
\hline $\begin{array}{l}\text { NYHA } 6 \mathrm{~m} \mathrm{FU} \\
\text { I } \\
\text { II } \\
\text { III } \\
\text { IV }\end{array}$ & $\begin{array}{l}10(25.64 \%) \\
22(56.41 \%) \\
6(15.38 \%) \\
1(2.56 \%)\end{array}$ & $\begin{array}{l}12(30.76 \%) \\
18(46.15 \%) \\
7(17.94 \%) \\
2(5.12 \%)\end{array}$ & \\
\hline \multirow[b]{2}{*}{$\begin{array}{l}\text { NYHA } 12 \mathrm{~m} \mathrm{~F} \\
\text { I } \\
\text { II } \\
\text { III } \\
\text { IV }\end{array}$} & $\begin{array}{l}\text { CABG } \\
\mathrm{N}=38\end{array}$ & $\begin{array}{l}\mathrm{PCI} \\
\mathrm{N}=38\end{array}$ & \multirow[b]{2}{*}{0.889} \\
\hline & $\begin{array}{l}16(42.10 \%) \\
18(47.36 \%) \\
4(10.52 \%) \\
0(0.00 \%)\end{array}$ & $\begin{array}{c}19(50.00 \%) \\
13(34.21 \%) \\
5(13.15 \%) \\
1(2.63 \%) \\
\end{array}$ & \\
\hline $\mathrm{P} 1$ & $<0.0001$ & $<0.0001$ & \\
\hline $\mathrm{P} 2$ & $<0.0001$ & $<0.0001$ & \\
\hline
\end{tabular}

*P1 compared $6 m$ to preprocedure. P2 compared $12 \mathrm{~m} F U$ to preprocedure. 
Revascularization of Left Anterior Descending Coronary Artery Chronic Total Occlusion by Percutaneous Coronary Intervention versus Coronary Artery Bypass Grafting (Early and Mid Term Outcome)

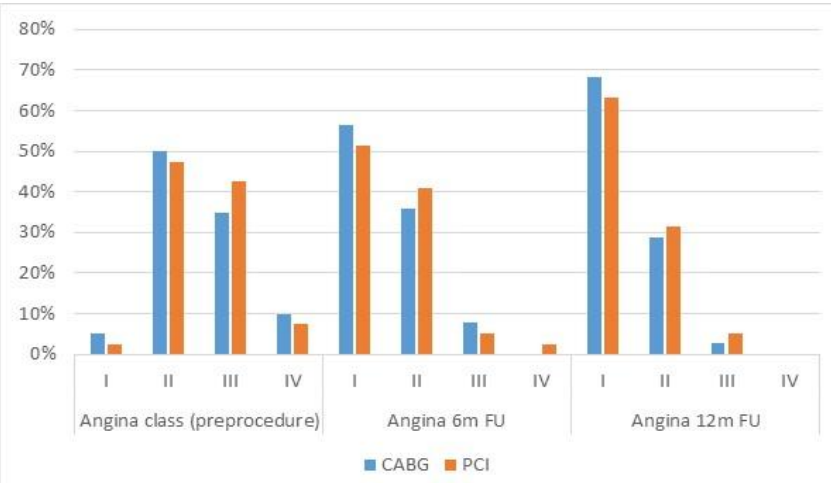

Fig1. Follow up angina class in both groups.

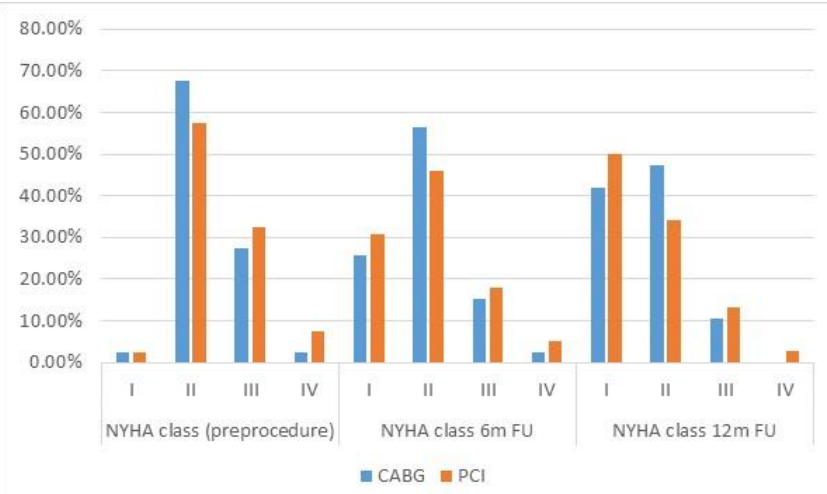

Fig2. Follow up NYHA class in both groups.

\subsection{Echocardiographic Follow Up Results}

\section{LV dimensions and EF}

There was a significant reduction of LVESD and LVEDD and improvement in systolic function EF in both groups with no statistically significant difference between two groups table $(7,8)$ and figure $(3)$.

Figures 4 represents one of our cases with CTO LAD revascularization through PCI with 2 DES.

Table7. Follow up LV systolic and diastolic dimensions in both groups

\begin{tabular}{|c|c|c|c|}
\hline Variables & CABG & PCI & $P$ value \\
\hline $\begin{array}{l}\text { LVESD }(\mathrm{mm}) \text { preprocedure } \\
\text { Mean } \pm \text { SD }\end{array}$ & $35.4 \pm 4.64$ & $34.05 \pm 5.652$ & 0.247 \\
\hline $\begin{array}{l}\text { LVESD }(\mathrm{mm}) 6 \mathrm{~m} \text { FU } \\
\text { Mean } \pm \text { SD }\end{array}$ & $34 \pm 4.224$ & $32.63 \pm 5.410$ & 0.209 \\
\hline \multirow[b]{2}{*}{$\begin{array}{l}\text { LVESD } 12 \mathrm{~m} \text { FU } \\
\text { Mean } \pm \text { SD }\end{array}$} & CABG & PCI & \multirow{4}{*}{0.343} \\
\hline & $32.67 \pm 3.912$ & $31.7 \pm 5.145$ & \\
\hline P1 & $<0.001$ & $<0.001$ & \\
\hline P2 & $<0.001$ & $<0.001$ & \\
\hline $\begin{array}{l}\text { LVEDD }(\mathrm{mm}) \text { preprocedure } \\
\text { Mean } \pm \text { SD }\end{array}$ & $52.85 \pm 4.423$ & $52.72 \pm 4.888$ & 0.905 \\
\hline \multirow[b]{2}{*}{$\begin{array}{l}\text { LVEDD } 6 \mathrm{~m} \text { FU } \\
\text { Mean } \pm S D\end{array}$} & CABG & PCI & \multirow[b]{2}{*}{0.858} \\
\hline & $51.25 \pm 4.1$ & $51.43 \pm 4.629$ & \\
\hline \multirow[b]{2}{*}{$\begin{array}{l}\text { LVEDD } 12 \mathrm{~m} \text { FU } \\
\text { Mean } \pm \text { SD }\end{array}$} & CABG & CABG & \multirow{4}{*}{0.835} \\
\hline & $49.9 \pm 3.901$ & $50.1 \pm 4.407$ & \\
\hline P1 & $<0.001$ & $<0.001$ & \\
\hline P2 & $<0.001$ & $<0.001$ & \\
\hline
\end{tabular}

$P 1$ compared $6 m$ to preprocedure.P2 compared $12 \mathrm{~m}$ to preprocedure. 
Dr Ahmed Boghdady et al.

Table8. Follow up EF in both groups
\begin{tabular}{|l|l|l|l|}
\hline Variables & CABG & PCI & P value \\
\hline $\begin{array}{l}\text { EF }(\%) \text { preprocedure } \\
\text { Mean } \pm \text { SD }\end{array}$ & $50.97 \pm 5.753$ & $51.75 \pm 7.615$ & 0.609 \\
\hline \multirow{2}{*}{$\begin{array}{l}\text { EF 6m FU } \\
\text { Mean } \pm \text { SD }\end{array}$} & CABG & PCI & \multirow{2}{*}{0.670} \\
\cline { 2 - 4 } & $52.68 \pm 5.618$ & $53.3 \pm 7.342$ & \\
EF 12m FU & CABG & PCI & 0.798 \\
\hline Mean \pm SD & $54.25 \pm 5.188$ & $54.6 \pm 6.861$ & \\
\hline P1 & $<0.001$ & $<0.001$ & \\
\hline P2 & $<0.001$ & $<0.001$ & \\
\hline
\end{tabular}

$P 1$ compared $6 m$ to preprocedure. $P 2$ compared $12 m$ to preprocedure.

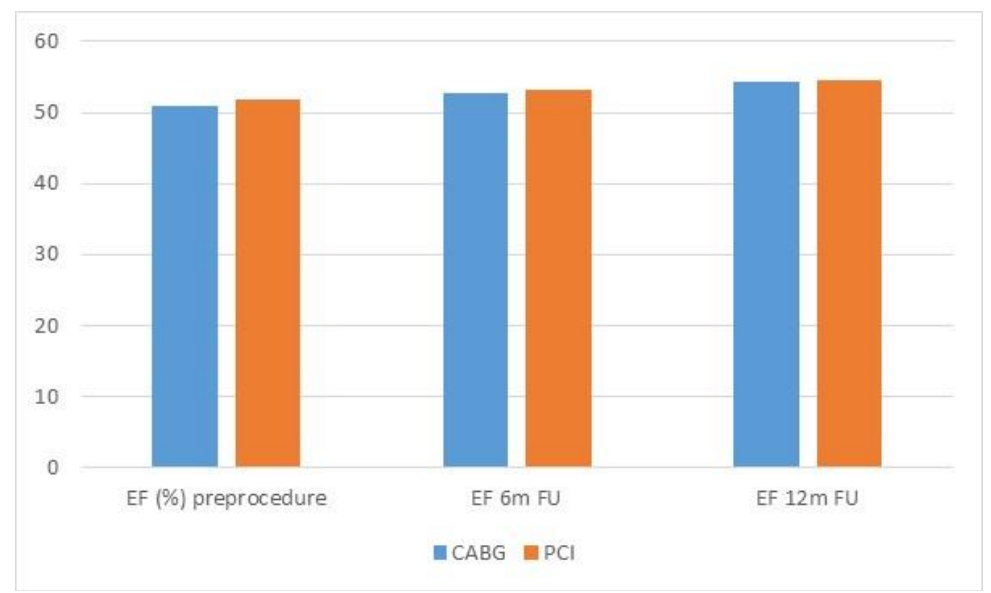

Fig3. Follow up EF in both groups.

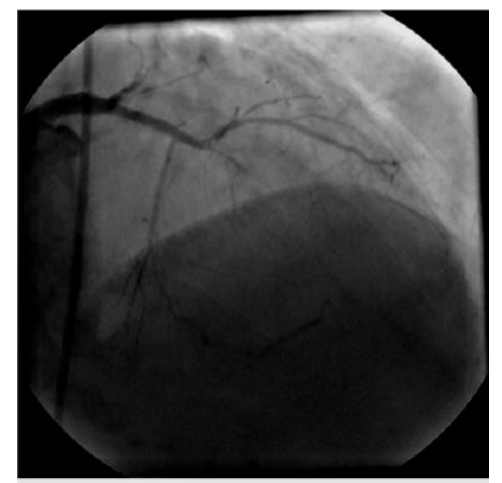

Fig4a. Coronary angiography showed CTO of $L A D$

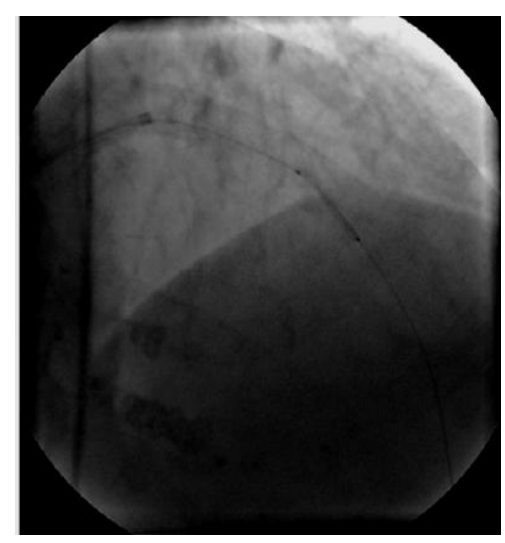

Fig4c. Dilatation of the lesion by $2 X 20 \mathrm{~mm}$ compliant balloon

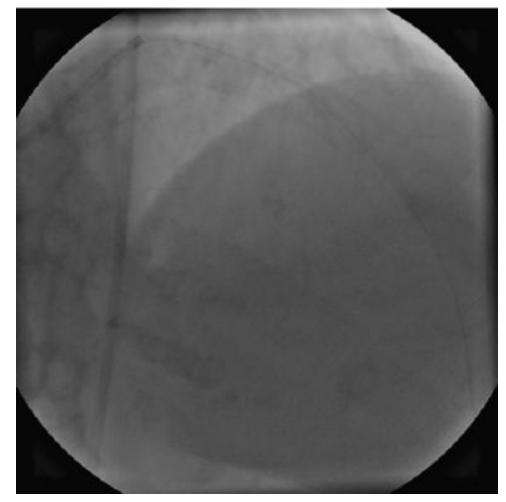

Fig4b. Showed wiring of LAD by PT2 moderate support hydrophilic wire

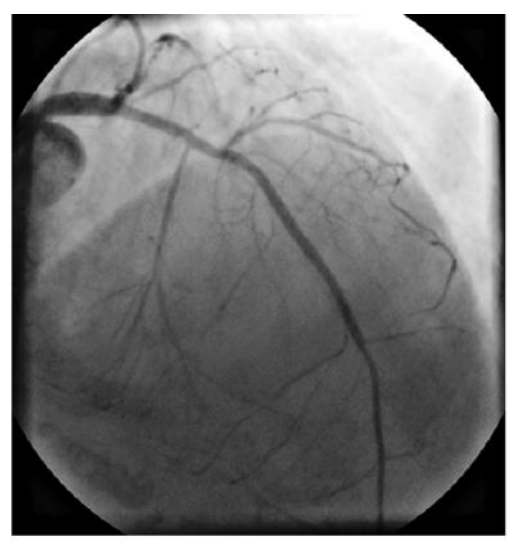

Fig4d. LAD after placement with 2 DES 
Revascularization of Left Anterior Descending Coronary Artery Chronic Total Occlusion by Percutaneous Coronary Intervention versus Coronary Artery Bypass Grafting (Early and Mid Term Outcome)

Major adverse cardiac events follow up results: Coronary angiography was repeated when indicated in 6 patients $(15 \%)$ in PCI group revealing significant lesions that need repeat revascularization in 5 patients $(12.5 \%$ ) 4 patients for LAD ( 3 patients with PCI and one patient with $\mathrm{CABG}$ ) and one patient for RCA treated by PCI. While in CABG group it was repeated in 3 patients (7.5\%) revealing significant lesion that need repeat revascularization through $\mathrm{PCI}$ in 2 patients $(5 \%)$ both in non LAD lesions (LCX and RCA).

\subsection{In Hospital MACE}

In CABG group 1 patient died during the $2^{\text {nd }}$ day after procedure due to occurrence of mediastinitis and multiorgan failure. In the PCI group, one patient died suddenly 6 hours after procedure due to ventricular fibrillation inspite attempt of resuscitation. One patients of the PCI group developed acute anterior MI following acute stent thrombosis 4 hours after the procedure and the patient underwent emergency coronary angiography and the thrombus is aspirated by thrombus aspiration catheter (Diver C) successfully. Acute MI occurred in one patient in the CABG group at the 2nd day post operative diagnosed by rising of serum cardiac troponins and ECG changes of Inferior MI, it was due to venous graft thrombosis and treated successfully by medical treatment. The patient didn't need repeat revascularization. Acute onset stroke occurred in one patient in CABG group at 3rd day post operative, presented as right hemiparesis diagnosed by CT brain (ischemic infarction) with partial improvement on medical therapy. No significant difference between both groups as regard occurrence of in hospital MACE; $\mathrm{P}=1.00$ as shown in Table (9)

\subsection{Follow up at 6 Months}

One patient in PCI group developed stroke 6 months after PCI manifested as complete hemiplegia diagnosed by CT brain as cerebral hemorrhage and treated with partial improvement of motor power. 1 patient in PCI group died 4 months after procedure due to cardiogenic shock. (This patient had baseline low EF 37\%). 2 patients in PCI had repeat revascularization, at 3 and 5 months after procedure due to development of UA to whom coronary angiography (CA) was done revealing significant instent restenosis ISR of LAD and treated by PCI with DES . No patients in CABG group needed repeat revascularization in 6 moths follow up as shown in Table (9)

\subsection{Follow up at 12 Months}

1 patient died 8 months after CABG by sudden death, 1 patient in PCI group died 4 months after procedure due to cardiogenic shock. (This patient had baseline low EF 37\%).1 patient in CABG group developed acute anterior MI 11 months after the procedure diagnosed by typical chest pain, raised ST segment in V1-3 with raised cardiac troponin level and thrombolytic therapy was given. Later CA revealed insignificant stenos is (30\%) in LIMA to LAD. 1 patient in PCI group developed acute anterior MI 11 months after PCI and coronary angiography revealed late stent thrombosis, attempt was done to aspirate instent thrombus but failed so the patient send for surgeons for CABG for repeat revascularization.1 patient in PCI group had repeat revascularization due to significant instent restenosis (ISR) occurred $8 \mathrm{~m}$ post PCI. The patient was treated by drug eluting balloon (Dior Paclitaxel eluting balloon) to RCA. 2 patients in CABG group needed repeat revascularization due to total occlusion occurred in SVG to LCX and RCA at 8 and 10 months post operative and was treated by DES to native LCX and RCA. Table (9)

\subsection{Over All MACE}

During the period of follow up, total MACE was more common in PCI group (10 patients, $25 \%$ ) than in CABG group (6 patients, $15 \%) ; \mathrm{P}=(0.043)$. No significant difference in death $(2[5 \%]$ in PCI vs 2 [5\%] in CABG), MI (2 [5\%] in PCI vs (1[2.5\%] in CABG) or stroke (1[2.5\%] in PCI vs $1[2.5 \%]$ in CABG) between both groups but repeat revascularization was non significantly higher in PCI group (5 patients, $12.5 \%$ ) than $\mathrm{CABG}$ group (2 patient, $5 \%$ ). $\mathrm{P}=0.083$ as shown in Table (9). All patients underwent either PCI or CABG within 0-26 days from coronary angiography. 
Dr Ahmed Boghdady et al.

Table9. Comparison between both groups as regard MACE

\begin{tabular}{|c|c|c|c|}
\hline Variables & CABG & PCI & $\mathrm{P}$ value \\
\hline $\begin{array}{l}\text { In hosp MACE } \\
\text {-Death } \\
\text {-MI } \\
\text {-Stroke } \\
\text {-TLR. }\end{array}$ & $\begin{array}{l}1(2.50 \%) \\
0(2.5 \%) \\
1(2.5 \%) \\
0(0 \%)\end{array}$ & $\begin{array}{l}1(2.5 \%) \\
1(2.5 \%) \\
0(2.5 \%) \\
1(2.5 \%)\end{array}$ & $\begin{array}{l}1.000 \\
0.323 \\
0.323 \\
0.323\end{array}$ \\
\hline \multirow[b]{2}{*}{$\begin{array}{l}\text { MACE 6m FU } \\
\text {-Death } \\
\text {-MI } \\
\text {-Stroke } \\
\text {-TLR. }\end{array}$} & CABG & PCI & \multirow[b]{2}{*}{$\begin{array}{l}0.323 \\
1.000 \\
0.323 \\
0.159\end{array}$} \\
\hline & $\begin{array}{l}0(0 \%) \\
0(0 \%) \\
0(0 \%) \\
0(0 \%)\end{array}$ & $\begin{array}{l}1(2.5 \%) \\
0(0 \%) \\
1(2.56 \%) \\
2(7.89 \%)\end{array}$ & \\
\hline \multirow[b]{2}{*}{$\begin{array}{l}\text { MACE 12m FU } \\
\text {-Death } \\
\text {-MI } \\
\text {-Stroke } \\
\text {-TLR }\end{array}$} & CABG & PCI & \multirow[b]{2}{*}{$\begin{array}{l}0.323 \\
1.000 \\
1.000 \\
1.000\end{array}$} \\
\hline & $\begin{array}{l}1(2.56 \%) \\
1(2.63 \%) \\
0(0 \%) \\
2(7.89 \%)\end{array}$ & $\begin{array}{l}0(0 \%) \\
1(2.63 \%) \\
0(0 \%) \\
2(7.89 \%)\end{array}$ & \\
\hline \multirow[b]{2}{*}{$\begin{array}{l}\text { - } \\
\text { Total MACE } \\
\text { - Total Death } \\
\text {-Total MI } \\
\text { - Total stroke } \\
\text {-Total TLR }\end{array}$} & $\begin{array}{l}\text { CABG } \\
\mathrm{N}=40\end{array}$ & $\begin{array}{l}\text { PCI } \\
\mathrm{N}=40\end{array}$ & \multirow[b]{2}{*}{$\begin{array}{l}0.0439 \\
1.000 \\
0.323 \\
1.000 \\
0.083\end{array}$} \\
\hline & $\begin{array}{l}(15 \%) \\
2(5 \%) \\
1(2.5 \%) \\
1(2.5 \%) \\
2(5 \%)\end{array}$ & $\begin{array}{l}10(25 \%) \\
2(5 \%) \\
2(5 \%) \\
1(2.5 \%) \\
5(12.5 \%)\end{array}$ & \\
\hline
\end{tabular}

\section{DisCUSSION}

Chronic total occlusion (CTO) of coronary arteries is the last stage of coronary artery atherosclerosis, accounting for one third of the disease confirmed by coronary angiography. 16 CTO exists in about $50 \%$ of patients with CAD, which is often accompanied by complex lesions, in about $15 \%$ patients as reported. This disease can result in myocardial ischemia, ventricular remodeling which lead to decreased myocardial contractile, reduction of quality of life and poor prognosis. 17

PCI is a therapeutic procedure used not only to recanalize vessels with total occlusion but also widely used in other aspects such as increase blood flow reperfusion, improve myocardial ischemia and left ventricular remodeling and decrease adverse cardiac events. $\mathbf{1 8 , 1 9}$

PCI for a CTO remains a major challenge in interventional cardiology, despite the notable advances of novel technologies and procedural techniques, including the use of DES. CTO is defined as an estimated $\geq 3$-month-old obstruction of a native coronary artery without any luminal continuity and TIMI grade 0 flow. 20

Although the revascularization decision is more straightforward in patients with CTO and LM/MV $\mathrm{CAD}$, patients with symptomatic isolated CTO represent a challenge for which revascularization decisions should be made. Medical treatment may not be sufficient to relieve symptoms or to improve long-term outcomes, and CABG may be seemed too invasive for single-vessel disease. Therefore, most patients will be referred for PCI. 21

PCI to CTO is now a well-accepted revascularization procedure. With average recanalization success rates of $>70 \%$ in experienced hands using contemporary CTO techniques, the presence of a CTO should not be a sufficient reason to switch from a percutaneous towards a surgical approach in MV $\mathrm{CAD}$ and the success rate is increased in selected patients with favorable angiographic criteria as short segment, presence of stump and absence of severe calcifications. ${ }^{22}$ Successful PCI for CTO has been shown to alleviate anginal symptoms, improve LVEF, decrease the need for CABG, and prolong life. 23

To the best of our knowledge, there are no trials comparing specifically outcomes following CABG or PCI in specifically LAD CTO patients. The SYNTAX trial assessed the optimal method of revascularization for patients with MV CAD of whom around one-quarter in each treatment arm had 
Revascularization of Left Anterior Descending Coronary Artery Chronic Total Occlusion by Percutaneous Coronary Intervention versus Coronary Artery Bypass Grafting (Early and Mid Term Outcome)

at least one CTO. $\mathbf{2 1}$ Overall, the trial found that as the complexity and extent of coronary disease increased (as assessed by the SYNTAX score), MACE rates increased at 3 years in patients treated with PCI compared with CABG. 24

The present study aims to compare the short \& intermediate term outcomes of successful revascularization of CTO of LAD by PCI and CABG.

The current study revealed no significant difference between both groups before revascularization as regard degree of angina class, NYHA class and echocardiographic data. All patients had CTO in LAD, with 11 patients (27.5\%) in PCI group had 2 VD versus 16 patients (40\%) in CABG group.

As regard symptoms relieve, current study revealed significant and comparable improvement in angina CCS class and NYHA class after revascularization of CTO by either PCI or CABG.

The results obtained were similar to those, achieved by Valenti et al, (2008) 25 and Prasad et al, (2007) 26 who reported improvement in symptoms post-PCI to CTO. Also, the presented data were analogical with the reported by Grantham, et al (2010) 27, Van Domburg et al, (2010) 28 and Abdallah et al, (2013) 29 who found that both stenting and CABG resulted in a significant improvement in angina class.

As regard improvement in LV function and dimensions, our results revealed significant improvement in LV dimensions and function after successful revascularization irrespective of the procedure performed either PCI or CABG.

Our results were similar to those reported recently by Uslu et al, (2013) 30 who showed a significant decrease in LVEDD, LVESD after PCI with significant increase in EF two months after the procedure. Similarly Nakamura et al, (2004) ${ }^{31}$ reported the results of a 5-center Asian registry in which 88 patients with successfully recanalized CTOs were treated with DES that ejection fraction significantly improved in these patients on follow up. Also our results were similar to those reported by Dzavik et al, (2001) 32 and Kirschbaum et al (2008) 33 who demonstrated statistically significant improvements in left ventricular function and regional wall motion with successful CTO recanalization.

According the presented results, there was a significant downgrading in MR severity after revascularization by either PCI or CABG without significant difference between both types of treatment.

The data obtained coincide with the results from 3 previous studies, indicating significant improvement in MR severity after CABG (Duarte et al, 1999; 34 Reyden et al, 2001; 35 and Kang et al $2006^{36}$ ).

Also the current findings were in agreement with Hickey et al, (1988) 37; Trichon et al, (2003) 38 ; and Ho et al, (2008) 39 who found a significant improvement in ischemic MR irrespective of the procedure performed either PCI or CABG. Similar results to ours were observed by Chua et al, (2010) 40 and Ammar et al, (2012) 41 who reported that successful total revascularization improve ischemic MR whether mild, moderate or severe and successful recanalization of CTO of coronary artery is associated with improvement in ischemic MR.

According the present investigation, there was no significant difference in incidence of mortality, MI and stroke between both groups at 1 year follow-up. The total MACE was higher in the PCI group than the CABG group and this was largely attributed to higher rate of repeat revascularization in the PCI patients than in CABG patients.

Prior information on relative long-term outcomes for $\mathrm{CABG}$ and stent implantation has been documented in many randomized, controlled trials with variable numbers of patients (123 to 1205 patients in each study). During follow-up periods ranging from one to three years, one of these studies found significantly lower mortality rates after CABG $\mathbf{4 2}$. On the other hand Goy et al, (2000) 43 found significantly higher mortality rates after CABG. 
Surrey et al, (2001) 44 included patients with single-vessel disease and found no significant difference in mortality rates between the two interventions. Hoffman et al, (2003) 45 reported in a meta-analysis of these studies that there were no significant differences in one-year or three-year mortality rates between both methods, but according those authors, patients who underwent CABG had significantly fewer subsequent revascularizations than patients who underwent stenting which coincides with our results.

Similar results to ours were reported by Serruys et al, (2006) 46 who revealed that at 5 years there was no difference in mortality, MI and stroke between patients treated with PCI and CABG. Overall MACE was higher in the stent group driven by the increased need for repeat revascularization. Also, Head et al, (2012) 24 reported that MACE rates increased at 3 years in patients treated with PCI compared with CABG attributed to higher rate of repeat revascularization. According Rodriguez et al, (2005) 47 and Hlatky et al, (2009) 48 reported that there was no difference in mortality and non fatal MI between stenting and surgery. Patients initially treated with CABG had better freedom from repeat interventions.

Our results were similar to those, achieved by Moses et al, (2003) 49 who reported that implantation of DES markedly enhance angiographic patency rates, freedom from recurrent angina, freedom from MACE and reduce the need for repeat revascularization after PCI in CTOs compared to BMS. Similar data were obtained with Mehran et al, (2011) $\mathbf{5 0}$ who found that the long-term MACE rate was significantly lower among patients treated with DES entirely due to a reduced need for TLR.

The presented results also coincided with those recently reported by Natasza et al, (2013) 51 who find that DES implantation during PCI for single CTO reduces MACE rate at 1-year and long-term followup due to the significant reduction of TLR in the DES group.

\section{STUdY LiMitations}

- Non randomization in choosing the type of revascularization procedure.

- Selection of patients with CTO either single vessel or 2 vessel disease with favorable angiographic characters for PCI.

- Relative small number of patients included in this study is a problem attributed to that it is a single centre study and to the special inclusion criteria made in this research to evaluate only LAD CTO revascularization.

- Studying the short and intermediate outcomes only.

- Inter and intra observer variability coefficients for the echocardiographic parameters reduced as possible but still to be considered.

- Large randomized clinical trials are needed to accurately assess and compare long term outcomes of treating patient with CTO with either PCI or CABG.

\section{Summary AND CONCLUSION}

Successful revascularization of LAD CTO by PCI is a relatively safe procedure and it revealed significant and comparable improvement in angina class and NYHA class in comparison with a more invasive procedure which is CABG. Also the results of current study showed significant and comparable improvement of LV dimensions, EF and degree of MR in both groups. On the other hand these data revealed no significant difference in mortality, MI and stroke in both groups, but the overall MACE was slightly higher in patients revascularized by PCI than in individuals underwent CABG due to higher rate of repeat revascularization in PCI group specially with good selection of patients with suitable angiographic criteria for attempt PCI. PCI to CTO is an effective therapeutic procedure with an acceptable high success rate \& low incidence of complications in patients with good selection criteria. Revascularization of CTO has beneficial clinical outcome. It alleviates anginal symptoms, improves NYHA class. Most patients become angina free after successful revascularization of CTO. Successful PCI to CTO improves global and segmental left ventricular systolic function. It improves ejection fraction \& LV dimensions with subsequent improvement in mitral regurgitation in symptomatic patients in short and intermediate follow-up. DES enhances angiographic patency rates, 
Revascularization of Left Anterior Descending Coronary Artery Chronic Total Occlusion by Percutaneous Coronary Intervention versus Coronary Artery Bypass Grafting (Early and Mid Term Outcome)

freedom from recurrent angina, and freedom from MACE. It reduces the need for repeat revascularization after PCI in CTOs compared to BMS. Treating CTO with DES is comparable with $\mathrm{CABG}$ in patients with favorable angiographic findings.

\section{RECOMMENDATIONS}

Good selection of patients with favorable angiographic characteristics of CTO to be treated by PCI enhance success rate and decrease the complications, need for CABG and overall MACE. The use of DES should be mandatory in all cases with PCI of CTO, the laws of health insurance and the state's expense treatment should be changed to sponsor DES for those patients specially who have CTO.

\section{REFERENCES}

[1] Andrew TL and Serruys BW (2006). Complete Revascularization Coronary Artery bypass Graft Surgery Versus Percutaneous Coronary Intervention. Circulation 114:249-255.

[2] Cuneo A and Tebbe U (2008). The management of chronic total coronary occlusions. Minerva Cardioangiol.56(5):527-41.

[3] Arif I, Callihan R and Helmi T (2008). Novel Use of Twin-Pass Catheter in Successful Recanalization of a Chronic Coronary Total Occlusion. The Journal of Invasive Cardiology. 20:1002-1004.

[4] Louvard Y and Lefèvre T (2008). Why perform PCI of coronary chronic occlusion and how? Ann Cardiol Angeiol (Paris).57(6):341-51.

[5] Drozd J, Wójcik J, Opalińska E, Zapolski T, Widomska-Czekajska T et al (2006). Percutaneous angioplasty of chronically occluded coronary arteries: long-term clinical follow-up. Kardiol Pol. 64(7):667-73; 674.

[6] Galassi AR, Tomasello SD, Costanzo L and Tamburino C (2008). Chronic total occlusions. J Ital cardiol. 9(10):666-73.

[7] Gottdiener JS, Bednarz J, Devereux R, Gardin J, Klein A, Manning WJ et al (2004). American Society of Echocardiography recommendations for use of echocardiography in clinical trials. $\mathbf{J}$ Am Soc Echocardiogr.17(10):1086-119.

[8] Lang RM (2006). Recommendations for chamber quantification. Eur J Echocardiogr. 7(2):79108.

[9] Rentrop KP, Cohen M, Blanke H and Phillips RA (1985). Changes in collateral channel filling immediately after controlled coronary artery occlusion by an angioplasty balloon in human subjects. JACC 5:587-592.

[10] Smith SC Jr, Feldman TE, Hirshfeld JW Jr, Jacobs AK, Kern MJ, King SB 3rd et al (2006). ACC/AHA/SCAI 2005 guideline update for percutaneous coronary intervention: A report of the American College of Cardiology/American Heart Association Task Force on practice guidelines (ACC/AHA/SCAI Writing Committee to update the 2001 Guidelines for Percutaneous Coronary Intervention). Circulation.113:e166 -286.

[11] Dangas GD, Claessen BE, Caixeta A, Sanidas EA, Mintz GS and Mehran R (2010). In-stent restenosis in the drug-eluting stent era. JACC. 56:1897.

[12] Cutlip DE, S Windecker, R Mehran, Boam A, Cohen DJ, van Es GA et al (2007). Clinical End Points in Coronary Stent Trials :A Case for Standardized Definitions. Circulation 115:23442351.

[13] Wijns W, Kolh P, Danchin N, Di Mario C, Falk V, Folliguet T et al (2010). Guidelines on myocardial revascularization. Eur Heart J 31:2501-2555.

[14] Thygesen K, Joseph SA, Harvey DW, Allan SJ, Hugo A K, Fred SA et al (2012). Expert Consensus Document. Third Universal Definition of Myocardial Infarction. JACC (23): 24272463.

[15] Beller GA and Ragosta M (2010). Decision making in multivessel coronary disease: the need for physiological lesion assessment. JACC Intv 3:315-317.

[16] Yamane M (2012). Current Percutaneous Recanalization of Coronary Chronic Total Occlusion, Rev Esp Cardiol 65(3):265-277. 
[17] Christofferson RD, Lehmann KG, Martin GV, Every N, Caldwell JH and Kapadia SR (2005). Effect of chronic total coronary occlusion on treatment strategy. Am J Cardiol 95, 1088-1091.

[18] Yu L, Gu T, Shi E and Jiang C (2012). Surgery for chronic total occlusion of the left main coronary artery. Ann Saudi Med 32:156-161.

[19] Hoye A (2012). Management of chronic total occlusion by percutaneous coronary intervention. Heart 98:822-828.

[20] Stone GW, Ellis SG, Cox DA, Hermiller J, O'Shaughnessy C, Mann JT et al (2004). A polymerbased paclitaxel-eluting stent in patients with coronary artery disease. N Engl J Med 350:221231.

[21] Serruys PW, Morice M-C, Kappetein P, Colombo A, Holmes DR, Mack MJ et al (2009). Percutaneous coronary intervention versus coronary-artery bypass grafting for severe coronary artery disease. N Engl J Med; 360:961-972.

[22] Carlo Di Mario N, Gerald S, Sianos G, Galassi AR, Büttner J, Dudek D et al (2007). European perspective in the recanalization of Chronic Total Occlusions (CTO): consensus document from the EuroCTO Club. EuroInterv 3:30-43.

[23] Chung CM, Nakamura S, Tanaka K, Tanigawa J, Kitano K, Akiyama T et al (2003). Effect of recanalization of chronic total occlusions on global and regional left ventricular function in patients with or without previous myocardial infarction. Catheter Cardiovasc Interv 60(3): 36874.

[24] Head SJ, Holmes DR, Mack MJ, Serruys PW, Mohr FW, Morice MC et al (2012). Risk profile and 3-year outcomes from the SYNTAX percutaneous coronary intervention and coronary artery bypass grafting nested registries. JACC Cardiovasc. Interv 5(6),618-625

[25] Valenti R, Migliorini A, Signorini U, Vergara R, Parodi G, Carrabba N et al (2008).Impact of complete revascularization with percutaneous coronary intervention on survival in patients with at least one chronic total occlusion. Eur Heart J; 29, 2336-2342.

[26] Prasad A, Rihal CS, Lennon RJ, Wiste HJ, Singh M and Holmes DR Jr (2007): Trends in outcomes after percutaneous coronary intervention for chronic total occlusions: a 25-year experience from the Mayo Clinic. JACC 49:1611-1618.

[27] Grantham JA, Jones PG, Cannon L and Spertus JA (2010). Quantifying the early health status benefits of successful chronic total occlusion recanalization: Results from the Flow- Cardia's Approach to Chronic Total Occlusion Recanalization (FACTOR) Trial. Circ Cardiovasc Qual Outcomes 3:284-290.

[28] Van Domburg RT, Daemen J, Morice MC, de Bruyne B, Colombo A, Macaya C et al (2010). Short- and long-term health related quality-of-life and anginal status of the Arterial Revascularization Therapies Study part II, ARTS-II; sirolimus-eluting stents for the treatment of patients with multivessel coronary artery disease. Euro Intervention. 5(8):962-7.

[29] Abdallah MS, Wang K, Magnuson EA, Spertus JA, Farkouh ME, Fuster V and Cohen DJ (2013). Quality of life after PCI vs CABG among patients with diabetes and multivessel coronary artery disease: a randomized clinical trial. JAMA 310(15):1581-1590.

[30] Uslu H, Cakmak N, Erkan ME, Hacımahmutoğlu S, Yilmaz S, Ozkan S and Sayar N (2013). Left ventricular remodeling assessment in patients with anterior acute myocardial infarction treated with successful primary percutaneous coronary intervention: an observational study .Anadolu Kardiyol Derg 13: 675-81.

[31] Nakamura S, Bae JH, Cahyadi YH, Udayachalerm W, Tresukosol D and Tansuphaswadikul S. (2008): Drug-Eluting Stents for the Treatment of Chronic Total Occlusion: A Comparison of Serial Angiographic Follow-Up with Sirolimus, Paclitaxel, Zotarolimus and Tacrolimus-Eluting Stent: Multicenter Registry in Asia. Circulation.118:S-737. Abstract.

[32] Dzavik V, Ghali WA, Norris C, Mitchell LB, Koshal A, Saunders LD et al (2001). Long-term survival in 11,661 patients with multivessel coronary artery disease in the era of stenting: a report from the Alberta Provincial Project for Outcome Assessment in Coronary Heart Disease (APPROACH) Investigators. Am Heart J 142:119-126.

[33] Kirschbaum SW, Baks T, Van denent M, Sianos G, Krestin GP, Serruys PW et al (2008).Evaluation of left ventricular function three years after percutaneous recanalization of chronic total coronary occlusions. Am J Cardiol 101:179-185. 
Revascularization of Left Anterior Descending Coronary Artery Chronic Total Occlusion by Percutaneous Coronary Intervention versus Coronary Artery Bypass Grafting (Early and Mid Term Outcome)

[34] Duarte IG, Shen Y, MacDonald MJ, Jones EL, Craver JM and Guyton RA (1999). Treatment of moderate mitral regurgitation and coronary disease by coronary bypass alone. Ann Thorac Surg. 68:426-430.

[35] Ryden T, Bech-H O, Brandrup-W G, Nilsson F, Svensson S and Jeppsson A (2001). The importance of grade 2 ischemic mitral regurgitation in coronary artery bypass grafting. Eur $\mathbf{J}$ Cardio-Thorac Surg 20:276-281.

[36] Kang D H, Kim MJ, Kang SJ, Song JM, Song H, Hong MK et al (2006). Mitral Valve Repair versus Revascularization Alone in the Treatment of Ischemic Mitral Regurgitation. Circulation 114:I-499-I-503.

[37] Hickey MS, Smith LR, Muhlbaier LH, Harrell FE Jr, Reves JG, Hinohara T et al (1988). Current prognosis of ischemic mitral regurgitation. Implications for future management. Circulation $78(3$ Pt 2):I51-9.

[38] Trichon BH, Glower DD, Shaw LK, Cabell CH, Anstrom KJ, Felker GM and O'Connor CM (2003). Survival after coronary revascularization, with and without mitral valve surgery, in patients with ischemic mitral regurgitation. Circulation 108(Suppl 2): II-103-110.

[39] Ho PC and Nguyen ME (2008). Multivessel coronary drug-eluting stenting alone in patients with significant ischemic mitral regurgitation: A 4-year follow up. J. Invasive Cardiol 20(1):41-3.

[40] Chua S, Hung J, Chung SY, Lin YC, Fu M, Wu CJ et al. (2010): Primary percutaneous coronary intervention lowers the incidence of ischemic mitral regurgitation in patients with acute STelevated myocardial infarction. Circulation. 74(11):2386-92.

[41] Ammar W, Mohamed A, and Salah H. (2012): Impact of Percutaneous Coronary Intervention on Ischemic Mitral Regurgitation. Heart Mirror Journal 6(3):264-268.

[42] SoS Investigators (2002). Coronary artery bypass surgery versus percutaneous coronary intervention with stent implantation in patients with multivessel coronary artery disease (the Stent or Surgery trial): a randomized controlled trial. Lancet 360:965-970.

[43] Goy JJ, Kaufmann U, Goy ED, Garachemani A, Hurni M, Carrel T et al (2000). A prospective randomized trial comparing stenting to internal mammary artery grafting for proximal, isolated de novo left anterior coronary artery stenosis: the SIMA trial. Mayo Clin Proc 75:1116-1123.

[44] Serruys PW, Unger F, Sousa JE, Jatene A, Bonnier HJ, Schönberger JP et al (2001).Comparison of coronary-artery bypass surgery and stenting for the treatment of multivessel disease. N Engl $\mathbf{J}$ Med 344:1117-1124.

[45] Hoffman SN, Ten Brook JA, Wolf MP, Pauker SG, Salem DN and Wong JB (2003). A metaanalysis of randomized controlled trials comparing coronary artery bypass graft with percutaneous transluminal coronary angioplasty: one- to eight-year outcomes. JACC41:12931304.

[46] Serruys PW, Kutryk MJ and Ong AT (2006). Coronary-artery stents. N Engl J Med 354: 483495.

[47] Rodriguez AE, Baldi J, Fernández Pereira C, Navia J, Rodriguez AM et al (2005). ERACI II Investigators. Five-year follow-up of the argentine randomized trial of coronary angioplasty with stenting versus coronary bypass surgery in patients with multiple vessel disease (ERACI II). JACC 46:582-588.

[48] Hlatky MA, Boothroyd DB, Bravata DM, Boersma E, Booth J, Brooks MM et al (2009). Coronary artery bypass surgery compared with percutaneous coronary interventions for multivessel disease: a collaborative analysis of individual patient data from ten randomised trials. Lancet 373: 1190-1197.

[49] Moses JW, Leon MB, Popma JJ, Fitzgerald PJ, Holmes DR, O'Shaughnessy C et al (2003). Sirolimus-eluting stents versus standard stents in patients with stenosis in a native coronary artery. N Engl J Med. 349:1315-1323.

[50] Mehran R, Claessen BE, Godino C, Dangas GD, Obunai K, Kanwal S et al (2011). On behalf of the Multinational Chronic Total Occlusion Registry Long-Term Outcome of Percutaneous Coronary Intervention for Chronic Total Occlusions JACC Intv 4:952-61). 
[51] Natasza G, Fijalkowski M, Jaguszewski M, Targonski R, Strozyk A, Cackowska M et al (2013). Major Adverse Cardiovascular Events After Drug-Eluting Stent Implantation in Patients With Single Chronic Total Occlusion: A Single-Center Registry. J Invasive Cardiol 25(11):567-572.

[52] Ong AT, Serruys PW, Mohr FW, Morice MC, Kappetein AP, Holmes DR Jr et al (2006). The SYNergy between percutaneous coronary intervention with TAXus and cardiac surgery (SYNTAX) study: design, rationale, and run-in phase. Am Heart J 151:1194-204.

[53] Daemen J, Kuck KH, Macaya C, LeGrand V, Vrolix M, Carrie D et al. (2008): Multivessel Coronary Revascularization in Patients with and without Diabetes Mellitus 3-Year Follow-Up of the ARTS-II Trial. JACC, 52, 1957-1967.

[54] Liu W, Ma CS, Kang JP, Du X, Chen F, Zhou YJ et al (2011). Comparison of drug eluting stent implantation with coronary artery bypass surgery in the treatment of patients with chronic total occlusion and multiple vessel disease. Chinese Medical Journal 124(8):1169-1174. 